\title{
Extraskeletal osteosarcoma of the thorax in a goat: case report
}

\author{
Ueli Braun ${ }^{1 *}$, Colin C Schwarzwald ${ }^{2}$, Eva Forster ${ }^{1}$, Mareike Becker-Birck ${ }^{1}$, Nicole Borel ${ }^{3}$ and Stefanie Ohlerth ${ }^{4}$
}

\begin{abstract}
Background: This report describes the results of clinical, ultrasonographic and computed tomographic examination of a 16-year-old goat with extraskeletal osteosarcoma of the thorax.

Case presentation: The lead clinical signs were abnormal condition and demeanour, fever, tachycardia, tachypnoea, dyspnoea and dilated jugular veins. Ultrasonographic examination of the thorax revealed a precardial mass, measuring 16.4 by 11.4 by $14.2 \mathrm{~cm}$. Computed tomographic examination showed dorsocaudal displacement of the trachea, heart and lungs to the right. A tentative diagnosis of mediastinal or pleural neoplasia was made, and the goat was euthanased and necropsied. A definitive diagnosis was based on histological examination of the mass.
\end{abstract}

Conclusions: To our knowledge, this case report is the first description of extraskeletal osteosarcoma of the thorax in goats and serves to broaden the diagnostic spectrum of thoracic diseases in this species. Extraskeletal osteosarcoma should be part of the differential diagnosis in goats with thoracic tumours.

\section{Background}

Tumours of the thorax are rare in goats [1]. Thymoma is the most common thoracic neoplasia in this species [2-6] and there are a few reports of lymphosarcoma and ovine pulmonary adenocarcinoma [1]. To the authors' knowledge, osteosarcoma has not been described in goats. Osteosarcoma occurs most commonly in the long bones and is rare in domestic animals other than cats and dogs [7]. Osteosarcoma may occur in organs other than the skeletal system, and the absence of a primary bone lesion is a prerequisite for the diagnosis of extraskeletal osteosarcoma. In dogs, extraskeletal osteosarcoma has been reported in the spleen, gastrointestinal tract, urogenital tract, liver, skin, mammary gland and subcutis [8-10]. A dog with osteosarcoma of the os penis was recently described [11]. Osteosarcoma commonly metastasizes in dogs, and haematogenous metastasis is more common than lymphogenous metastasis [9]. The major target organs include the lungs, liver, mediastinum, omentum and heart [8]. There have been two reports on osteosarcoma in goats; one case involved

\footnotetext{
* Correspondence: ubraun@vetclinics.uzh.ch

'Department of Farm Animals, Vetsuisse Faculty, University of Zurich, Zurich, Switzerland

Full list of author information is available at the end of the article
}

osteosarcoma of the humerus in a Toggenburg goat [12] and the other described osteosarcoma of the metacarpus of a seven-year-old Alpine goat [13]. The purpose of this report was to describe the clinical, ultrasonographic and computed tomographic findings in a goat with extraskeletal osteosarcoma.

\section{Case presentation}

A 16-year-old castrated male miniature goat was referred to our clinic because of poor appetite, tachypnoea and tachycardia of several weeks duration. The general condition and demeanour were abnormal, the rectal temperature was $40.2^{\circ} \mathrm{C}$ (normal, $38.1-40.0^{\circ} \mathrm{C}$, [14]), the heart rate was 160 beats per minute (normal, 70 - 90 beats per minute, [14]) and there was a mild arrhythmia. Both jugular veins were distended. The goat had dyspnoea, abdominal respiratory effort and a respiratory rate of 60 breaths per minute (normal, 15 30 breaths per minute, [14]). Auscultation of the lungs revealed increased respiratory sounds. Examination of the intestinal and urinary tracts, musculoskeletal system and central nervous system yielded no abnormal findings.

Based on reference values established in this clinic [15], the activities of glutamate dehydrogenase (103 U/l, 
normal 3.1 - $19.8 \mathrm{U} / \mathrm{l}$ ) and sorbitol dehydrogenase (128 $\mathrm{U} / \mathrm{l}$, normal 20.4 - $68.7 \mathrm{U} / \mathrm{l}$ ) were increased and the serum concentrations of magnesium $(0.64 \mathrm{mmol} / \mathrm{l}$, normal $0.9-1.4 \mathrm{mmol} / \mathrm{l})$ and inorganic phosphate $(0.85$ $\mathrm{mmol} / \mathrm{l}$, normal $1.2-2.9 \mathrm{mmol} / \mathrm{l}$ ) were decreased. Other biochemical variables and a complete blood cell count were within normal limits.

Ultrasonographic examination of the thorax and heart using a phased-array sector probe (1.9/4.0 $\mathrm{MHz}$ octave harmonics, GE Vivid 7 Dimension, GE Medical Systems, Glattbrugg, Switzerland) revealed a multi-chambered precardial mass measuring $11 \mathrm{~cm}$ in diameter (Figure 1) and mild pleural and pericardial effusion. Electrocardiography showed ventricular extrasystoles, indicating secondary lesions of the myocardium. Subjectively, the right ventricle appeared enlarged compared to the left ventricle, but no obvious structural changes of the heart were detected during echocardiographic examination.

A non-contrast computed tomographic (CT) examination of the thorax as well as CT angiography were carried out using a 40-slice CT scanner (Somatom Sensation Open, Siemens, Erlangen, Germany). General anaesthesia was induced and the goat was positioned in sternal recumbency and stabilized with multiple foam blocks. Transverse contiguous slices were obtained from the thoracic inlet to the cranial abdomen. Technical settings were $120 \mathrm{KV}, 200 \mathrm{~mA}, 1 \mathrm{~s}$ tube rotation, $5 \mathrm{~mm}$ slice collimation and a pitch of 1.0 and 0.8 for the noncontrast scan and $\mathrm{CT}$ angiography, respectively. The data were reconstructed to an image series with $1.5 \mathrm{~mm}$ slice thickness using a medium-frequency image reconstruction algorithm (soft tissue) and a high-frequency image reconstruction algorithm (bone), respectively. CT

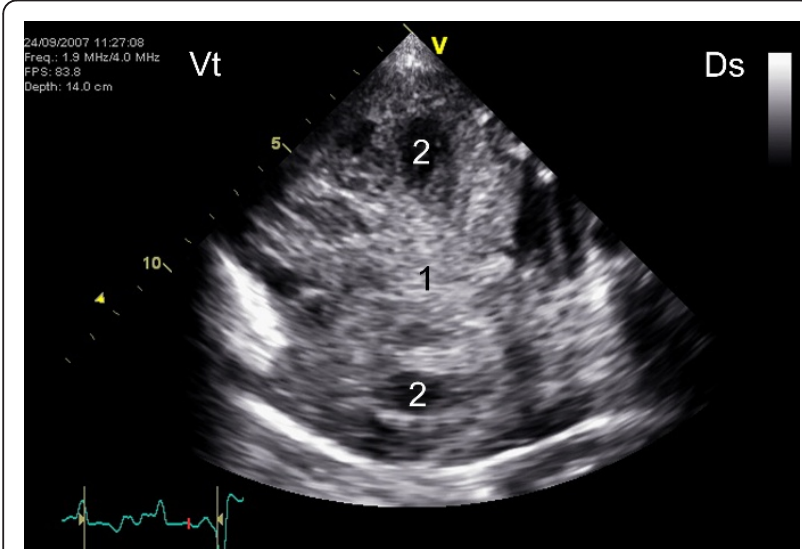

Figure 1 Ultrasonogram of osteosarcoma. Ultrasonographic appearance of a thoracic precardial mass in a miniature goat. The mass was round and multicystic and had a diameter of approximately $11 \mathrm{~cm}$. A sector scanner with a phased-array probe and a frequency of 1.9 to $4.0 \mathrm{MHz}$ (octave harmonics) was used. 1 Mass, 2 Cyst, Ds Dorsal, Vt Ventral. angiography was carried out according to a recently published protocol [16]. CT images were transferred to a work station and reviewed with dedicated software (OsiriX Open Source ${ }^{\mathrm{TM}}$ Version 3.2.1, OsiriX Foundation, Geneva, Switzerland). The precardial mass, which was located on the ventral aspect of the thorax and measured $16.4 \times 11.4 \times 14.2 \mathrm{~cm}$, extended from the thoracic inlet to the diaphragm (Figure 2). There was marked displacement of the heart and trachea dorsocaudally and to the right. The lungs and bronchi were compressed and displaced dorsally as well as caudally. The ventral aspects of the lungs had areas with moderate consolidation and air bronchograms, particulary of the right side. Intra- and interlobular septa as well as ,ground-glass'-like opacities, air bronchograms and multiple indentations in the pulmonary pleura were seen caudodorsally on both sides. The precardial mass was hypodense centrally and isodense peripherally relative to the surrounding soft tissues, and there were mineralized areas ventrally. The accumulation of contrast medium after intravenous administration $(2 \mathrm{ml} / \mathrm{kg}$ body weight, infusion rate $4 \mathrm{ml} / \mathrm{sec}$; Ultravist ${ }^{\circledR}-300$, Schering, Germany) was limited to peripheral areas of the mass and to small nodules between the mass, the heart and the thoracic wall. The thoracic wall appeared normal. Based on the CT examination, a diagnosis of mediastinal or pleural neoplasia and moderate multilobar pneumonia was made. An abscess was unlikely because of the additional nodules identified by the contrast medium.

Because the prognosis was grave, the goat was euthanased and a post-mortem examination was carried out.

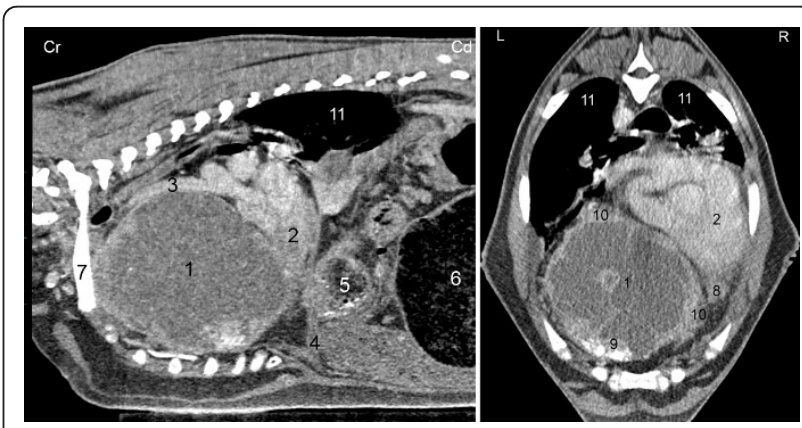

Figure 2 CT image of thoracic osteosarcoma. Computed tomographic soft-tissue window after intravenous application of contrast medium in a miniature goat with thoracic osteosarcoma. Sagittal section of the right hemithorax (A) and transverse section at the level of the 8 th thoracic vertebra (B). The mass extended from the thoracic inlet to the diaphragm and displaced the heart dorsally and to the right. Accumulation of contrast medium was limited to the periphery of the mass. A smaller nodular mass was seen ventral to the heart. 1 Precardial mass, 2 Heart, 3 Truncus brachiocephalicus, 4 Diaphragm, 5 Reticulum, 6 Rumen, 7 First rib, 8 Nodular mass, 9 Amorphous mineralization, 10 Peripheral accumulation of contrast medium, 11 Lung, Cr Cranial, Cd Caudal, L Left, R Right. 
Examination of the thorax revealed a large firm white oval mass cranial to the heart (Figure 3). The mass displaced the heart to the right side of the thorax and resulted in compression of the heart, trachea and lungs. The cut surface of the mass had cystic lesions and a central area of necrosis. Adhesions between the mass and the pericardium and sternum were seen in the cranial thorax, and there were multiple nodules ranging in diameter from 0.5 to $1.0 \mathrm{~cm}$ in the right pleural space. Several contact metastases, which were not connected directly to the ribs, were observed on the costal pleura. The ribs had no macroscopic lesions. Histological examination of the mass and neoplastic nodules revealed irregular osteoid islets, which were surrounded and occasionally infiltrated by malignant osteoblasts (Figure 4). The neoplastic osteoblasts were oval, elongated or spindle-shaped, and the nuclei were generally pleomorphic and often eccentrically placed. There were a few mitotic figures, and a large necrotic area was seen in the centre of the tumour. The definitive diagnosis was thoracic osteosarcoma with contact metastases on the costal pleura and diaphragm. In addition, there was severe acute pulmonary oedema with severe purulent pneumonia in the ventral lung lobes (presumably caused by aspiration) and mild verminous pneumonia in the diaphragmatic lung lobes.

The lead clinical signs in this patient were bilateral distension of the jugular veins, tachycardia and tachypnoea. Distension of the jugular veins primarily suggests right-sided cardiac insufficiency, but in the absence of cardiac signs, may indicate obstruction of the cranial vena cava by a thrombus or compression of the vein by an abscess, tumour or thoracic effusion [17]. In our patient, right-sided cardiac insufficiency could not be ruled out because of the tachycardia and arrhythmia and the right ventricle appeared enlarged

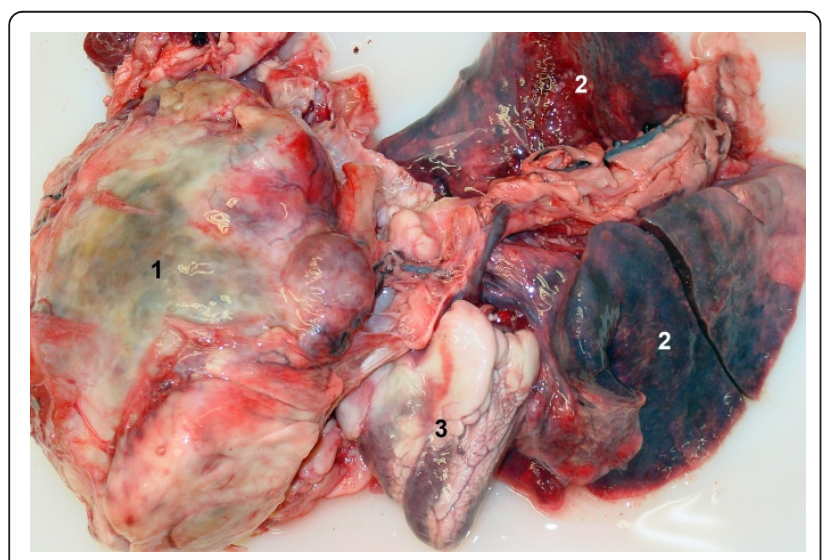

Figure 3 Gross pathological findings. Gross pathological findings in a miniature goat with thoracic osteosarcoma. 1 Precardial mass, 2 Lung, 3 Heart.

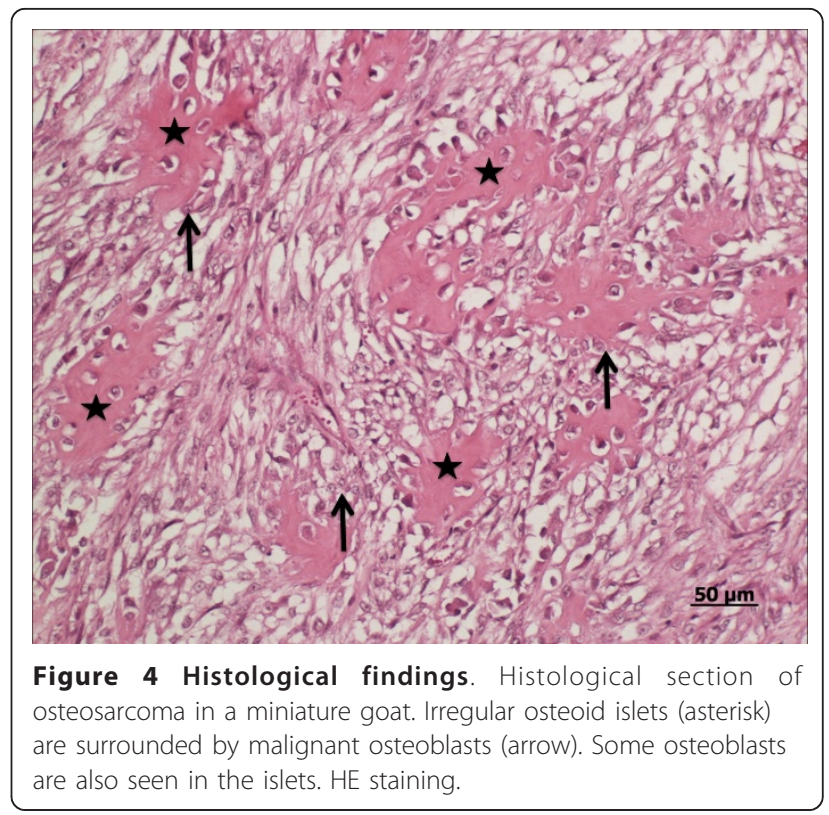

on ultrasonograms. However, a combination of ultrasonography, radiography and $\mathrm{CT}$ allowed a tentative diagnosis of a tumour. The CT exam provided detailed images of the thoracic organs, which could not be accomplished with ultrasonography. A recent CT study of the thorax in 26 healthy goats [18] was very helpful for interpreting the lesions in our patient. An abscess was not part of the differential diagnosis because the total leukocyte count and concentrations of fibrinogen and total solids were within the reference ranges. The advanced age of our patient favoured a diagnosis of neoplasia. Differential diagnoses for the thoracic tumour included thymoma, thymic lymphoma [1], metastatic carcinoma and tumour of the aortic bodies. Thymic lymphoma tends to occur in younger animals, whereas thymoma has been reported more often in adult or aged animals [19]. Furthermore, regional lymph nodes, which were normal in our patient, are commonly involved in thymic lymphoma, but not in thymoma. A fine needle aspirate or ultrasound-guided biopsy of the mass was not carried out because of the grave prognosis and advanced age of the patient. Instead, a postmortem examination served to provide a definitive diagnosis. Because there were no indications of skeletal involvement, the osteosarcoma was considered extraskeletal. Extraskeletal osteosarcoma has been described in dogs, primarily in the mammary gland, spleen, gastrointestinal and urogenital tracts, liver, skin and subcutis [10]. However, to our knowledge, this tumour has not been reported in domestic animals. Interestingly, a pleural osteosarcoma with pulmonary involvement was recently described in the human literature [20]. 


\section{Conclusion}

The differential diagnosis in goats with distended jugular veins, tachycardia and tachypnoea should include abscess and neoplasia in addition to right-sided heart failure. Ultrasonography, radiography and computed tomography are useful for diagnosing neoplasia and determining the extent of the lesions. To our knowledge, this case report is the first description of extraskeletal osteosarcoma of the thorax in goats and serves to broaden the diagnostic spectrum of thoracic disease in this species. Extraskeletal osteosarcoma should be part of the differential diagnosis in goats with thoracic neoplasia.

\section{Consent}

Consent was obtained from the owner of the goat for publication of this case report and any accompanying images.

\section{Acknowledgements \\ The authors thank Dr Rolf Kamber for referring the goat to our clinic, the technicians of the Medical Laboratory for the haematological and biochemical analyses and the animal assistants for looking after the goat.}

\section{Author details}

${ }^{1}$ Department of Farm Animals, Vetsuisse Faculty, University of Zurich, Zurich, Switzerland. ${ }^{2}$ Department of Horses, Vetsuisse Faculty, University of Zurich, Zurich, Switzerland. ${ }^{3}$ Institute of Veterinary Pathology, Vetsuisse Faculty, University of Zurich, Zurich, Switzerland. ${ }^{4}$ Diagnostic Imaging Section, University of Zurich, Zurich, Switzerland.

\section{Authors' contributions}

UB prepared the manuscript and supervised the clinical examination, CS performed the ultrasonographic examination, EF examined the goat, MBB contributed to the clinical research, NP performed the postmortem examination, SO was responsible for the $\mathrm{CT}$ examination. All authors have read and approved the manuscript.

Received: 18 October 2010 Accepted: 19 September 2011

Published: 19 September 2011

\section{References}

1. Smith MC, Sherman DM: Respiratory system. In Goat Medicine.. 2 edition. Edited by: Smith MC, Sherman DM. Ames: Wiley-Blackwell; 2009:339-376.

2. Hadlow WJ: High prevalence of thymoma in the dairy goat. Report of seventeen cases. Vet Pathol 1978, 15:153-169.

3. Rostkowski CM, Stirtzinger T, Baird JD: Congestive heart failure associated with thymoma in two Nubian goats. Can Vet J 1985, 26:267-269.

4. Parish SM, Middleton JR, Baldwin TJ: Clinical megaoesophagus in a goat with thymoma. Vet Rec 1996, 139:94.

5. Olchowy TW, Toal RL, Brenneman KA, Slauson DO, McEntee MF: Metastatic thymoma in a goat. Can Vet J 1996, 37:165-167.

6. Braun U, Steininger $K$, Irmer M, Hagen R, Ohlerth S, Ruhl S, Ossent P: Ultraschall- und CT-Befunde bei einer Ziege mit Thymom im Mediastinum. Schweiz Arch Tierheilk 2009, 151:332-335.

7. Thompson K: Osteosarcoma. In Jubb, Kennedy, and Palmer's Pathology of Domestic Animals.. Fifth edition. Edited by: Maxie GM. Edinburgh: Saunders Elsevier; 2007:112-118.

8. Kuntz CA, Dernell WS, Powers BE, Withrow S: Extraskeletal osteosarcomas in dogs: 14 cases. J Am Anim Hosp Assoc 1998, 34:26-30.

9. Langenbach A, Anderson MA, Dambach DM, Sorenmo KU, Shofer FD: Extraskeletal osteosarcomas in dogs: a retrospective study of 169 cases (1986-1996). J Am Anim Hosp Assoc 1998, 34:113-120.
10. Chun R, de Lorimier LP: Update on the biology and management of canine osteosarcoma. Vet Clin Small Anim 2003, 33:491-516.

11. Peppler C, Weissert D, Kappe E, Klump S, Kramer M, Reinacher M, Neiger R: Osteosarcoma of the penile bone (os penis) in a dog. Aust Vet J 2009, 87:52-55.

12. Steinberg $H$, George C: Fracture-associated osteogenic sarcome and mandibular osteoma in two goats. J Comp Path 1989, 100:453-457.

13. Román-Muñiz IN, Van Metre DC, Cruz-Arámbulo R, de J, Basaraba RJ, Callan RJ, Withrow SJ: What is your diagnosis? Cortical lysis, medullary sclerosis, and periosteal bony proliferation of the distal portion of the metacarpus. J Am Vet Med Assoc 2003, 222:717-718.

14. Diffay BC, McKenzie D, Wolf C, Pugh DG: Handling and examination of sheep and goats. In Sheep and Goat Medicine. Edited by: Pugh DG. Philadelphia: WB Saunders Company; 2002:1-17.

15. Tschuor AC, Riond B, Braun U, Lutz H: Hämatologische und klinischchemische Referenzwerte für adulte Ziegen und Schafe. Schweiz Arch Tierheilk 2008, 150:287-295

16. Makara M, Dennler M, Kühn K, Kalchofner K, Kircher P: Effect of contrast medium injection duration on peak enhancement and time to peak enhancement of canine pulmonary arteries. Vet Radiol and Ultrasound published online: 10 AUG 2011

17. Braun U, Lejeune B, Schweizer G, Puorger M, Ehrensperger F: Clinical findings in 28 cattle with traumatic pericarditis. Vet Rec 2007, 161:558-563.

18. Ohlerth S, Becker-Birck M, Augsburger H, Jud R, Makara M, Braun U: Computed tomography measurements of thoracic structures in 26 clinically normal goats. Res Vet Sci 2011, http://dx.doi.org/10.1016/j. rvsc.2010.10.019 PMID: 21071049.

19. Jacobs RM, Messick JB, Valli VE: Thymoma. In Tumors in Domestic Animals. Edited by: Meuten DJ. Ames: lowa State Press; 2002:165-166.

20. Matono R, Maruyama R, Ide S, Kitagawa D, Tanaka J, Saeki H, Shimokama T, Higashi H: Extraskeletal osteosarcoma of the pleura: a case report. Gen Thorac Cardiovasc Surg 2008, 56:180-182.

doi:10.1186/1746-6148-7-55

Cite this article as: Braun et al.: Extraskeletal osteosarcoma of the thorax in a goat: case report. BMC Veterinary Research 2011 7:55.

\section{Submit your next manuscript to BioMed Central and take full advantage of:}

- Convenient online submission

- Thorough peer review

- No space constraints or color figure charges

- Immediate publication on acceptance

- Inclusion in PubMed, CAS, Scopus and Google Scholar

- Research which is freely available for redistribution 